\title{
Role of Prefrontal Cortex in Conscious Visual Perception
}

\author{
Camilo Libedinsky and Margaret Livingstone \\ Department of Neurobiology, Harvard Medical School, Boston, Massachusetts 02115
}

\begin{abstract}
Early visual areas are required for conscious visual perception, but recent evidence suggests that parts of the frontal lobe might also play a key role. However, it remains unclear whether frontal brain areas are involved in visual perception or merely use information from visual regions to drive behavior. One such frontal cortical area, the frontal-eye field (FEF), has been shown to have fast visual responses, thought to reflect mostly low-level visual processing, and delayed responses that correlate with perceptual reports. The latter observation is consistent with the idea that FEF uses visual information from (slower) visual regions to guide behavior. Here we ask whether fast visual responses in FEF also carry information related to the perceptual state of animals. We recorded single-cell activity in two monkeys trained to report the presence or absence of a visual target under conditions that evoke the illusory disappearance of the target (motion-induced blindness). We found that fast responses in FEF strongly correlated with the perceptual report of the animal. It is unlikely that short-latency perceptually correlated activity is inherited from early visual areas, since response latencies in FEF are shorter than those of visual areas with perceptually correlated activity. These results suggest that frontal brain areas are involved in generating the contents of visual perception.
\end{abstract}

\section{Introduction}

When a visual stimulus impinges upon the retina, a series of events ensue that may or may not lead to the conscious perception of the stimulus. Imaging and neurophysiological studies have shown that stimuli that evoke massive changes in brain activation across several brain regions may nevertheless remain unseen (Logothetis and Schall, 1989; Leopold and Logothetis, 1996; Lumer et al., 1998; Thompson and Schall, 1999; Donner et al., 2008; Libedinsky et al., 2009; Schölvinck and Rees, 2010). Why some patterns of brain activity lead to conscious perception and others do not is not understood. One way to approach this question is to compare brain activity when a visual stimulus is perceived to when it is not perceived. Under normal viewing conditions, several posterior cortical areas in the occipital, temporal, and parietal lobes respond to specific attributes of visual stimuli; they are thus known as visual cortical areas (henceforth referred to as early visual areas). However, under special viewing conditions where the visual stimulus can be dissociated from the perceptual state (visual illusions), activity in some areas of visual cortex correlates with the stimulus being presented (generally early visual areas), while activity in other areas correlates with the perceptual state of the subject (generally later visual areas) (Logothetis and Schall, 1989; Leopold and Logothetis, 1996; Sheinberg and Logothetis, 1997; Lumer et al., 1998; Libedinsky et al., 2009).

Areas in the frontal lobe of the brain are thought to receive and use perceptual information from various sensory areas to guide cognition and behavior. For example, the frontal-eye field (FEF) in the frontal lobe receives direct connections from extrastriate visual areas and in turn sends projections to oculomotor struc-

\footnotetext{
Received July 13, 2010; revised Sept. 9, 2010; accepted 0ct. 14, 2010.

We thank Richard Born, John Maunsell and John Assad for their helpful comments and support.

Correspondence should be addressed to Camilo Libedinsky, 220 Longwood Avenue, WAB 232, Boston, MA 02115.

E-mail: libedinsky@post.harvard.edu.

DOI:10.1523/JNEUROSCI.3620-10.2011

Copyright $\odot 2011$ the authors $\quad 0270-6474 / 11 / 310064-06 \$ 15.00 / 0$
}

tures controlling eye movements (Schall, 2002). The latency of this sensorimotor transformation can be as fast as $130 \mathrm{~ms}$ (Thompson et al., 1996; Schmolesky et al., 1998). A population of FEF neurons respond to visual stimuli at latencies of $<100 \mathrm{~ms}$ (Bruce and Goldberg, 1985; Pouget et al., 2005; Kirchner et al., 2009). Previous studies using visual illusions have shown that these short latency visual responses in FEF correlate with the visual stimulus, albeit with a small bias predictive of perceptual state (Thompson and Schall, 1999), whereas a second response, delayed by $\sim 50 \mathrm{~ms}$, correlates with the perceptual report regardless of the visual stimulus (Thompson and Schall, 2000). It is unclear whether this late perceptually correlated activity originates in FEF or is conveyed to FEF by other cortical areas. Since activity in most visual areas precedes this late FEF response, including areas with perceptually correlated activity that project to FEF, it is likely that the late perceptually correlated activity observed in FEF is inherited from visual areas. If so, FEF would not be involved in generating the contents of visual perception, but only in receiving visual activity from visual areas and using it to guide behavior and cognition.

In the present study, we challenge this view by providing evidence that the early visual responses in FEF neurons also strongly correlate with perceptual state. Given the short latencies of these initial responses, it is unlikely that this perceptually correlated activity is inherited from early visual areas, suggesting that FEF may be causally involved in visual perception.

\section{Materials and Methods}

Behavioral methods. When a highly salient visual target is surrounded by moving objects in nonoverlapping close proximity, the target becomes intermittently invisible to the observer, even though it is physically continuously present. This phenomenon is known as motion-induced blindness (MIB) (Bonneh et al., 2001).

Two male rhesus monkeys, Macaca mulatta, were trained in this MIB task. They were required to fixate on a small spot $\left(0.5^{\circ}\right)$ while reporting the appearance or disappearance of a target, located $7^{\circ}$ to the left of the fixation spot, using a lever press. This target location was chosen to optimize the population response of the cells recorded in our chronic 
electrode array. The target, a yellow circle $0.3^{\circ}$ in radius and a luminance of $111 \mathrm{~cd} / \mathrm{m}^{2}$, was surrounded by mask that consisted of an array of 81 $(9 \times 9)$ blue crosses with a luminance of $19.4 \mathrm{~cd} / \mathrm{m}^{2}$ forming a square of $10^{\circ} \times 10^{\circ}$ (supplemental Fig. S1, available at www.jneurosci.org as supplemental material). Each trial lasted between 10 and $15 \mathrm{~s}$, at the end of which a juice reward was given if the target transitions had been correctly reported within $1 \mathrm{~s}$ of each transition throughout the trial. Trials were aborted and a $3 \mathrm{~s}$ time-out period occurred if the animal broke fixation, failed to report a target transition, or reported the wrong transition in any trial. During recording sessions, in $70 \%$ of the trials the target was turned $\mathrm{ON}$ and $\mathrm{OFF}$ at a rate of $1.5-4.5 \mathrm{~s}$. This eliminates illusory disappearances in humans. In $30 \%$ of the trials, monkeys were presented with a prolonged period of target $\mathrm{ON}(8-10 \mathrm{~s})$. In humans, this condition induces perceptual disappearances; i.e., MIB. Trial types were intermixed and not cued. During the prolonged target ON period, the monkey was required to maintain fixation but was not punished or rewarded for reporting changes in the target. If the monkeys reported a disappearance or reappearance of the target during this long target $\mathrm{ON}$ period, the trial was not aborted. We therefore refer to the transitions occurring during long target $\mathrm{ON}$ periods as illusory transitions, and transitions due to physical changes of the target as real transitions. The monkeys had no incentive to pull the lever during this period other than accurately reporting their perceptual state, since reward was only given at the end of the $15 \mathrm{~s}$ trial. All trials ended with the target disappearing and reappearing to ensure that the monkeys were faithfully reporting their perceptual state at the end of each trial.

Eye position was monitored with an infrared eye tracking system (ISCAN) at $250 \mathrm{~Hz}$ in both animals and smoothed with an adaptive median filter (seven frames). The spatial resolution of the eye tracker was not fine enough to detect microsaccades; however, we were able to reliably identify small eye-movements within the fixation window between 0.5 and 1 degree. Small eye-movements were detected using a velocity threshold set at four times the SD of the signal derived from the fixation period (Asaad et al., 2000).

Recording methods. Recordings were made from the right hemisphere FEF of the two trained monkeys using $32 \mathrm{Pt} / \mathrm{Ir}$ electrodes implanted under the dura mater of each monkey. Each array (FMA; Microprobe) consisted of 16 electrodes (impedance, 0.5-1.5 M $\Omega$ ); two arrays were implanted in the FEF of each monkey. FEF was identified by the cortical sulcal pattern during implantation surgery. The arrays were implanted in the anterior bank of the arcuate sulcus and their location in FEF was later verified using microstimulation to evoke eye movements. Biphasic pulse trains ( $100 \mathrm{~ms}$ in duration, pulse duration $200 \mu \mathrm{s}, 200 \mathrm{~Hz}$ ) were delivered through each microelectrode. Sites where stimulation of $50 \mu \mathrm{A}$ or lower elicited eye movements at least $50 \%$ of the time, plus regions within 2-3 $\mathrm{mm}$ of these locations, were considered to be in FEF. All sites recorded and reported herein met these criteria. The electrode arrays were implanted in fully trained animals. The results reported here were collected within a period of 3-4 months after the electrodes were implanted. Recording sessions began between 2 and 3 weeks after the implantation. All cells reported here remained well isolated throughout the length of the recording session. Neural waveforms were stored, digitalized, and sorted offline using principal components (Plexon Systems).

Although the electrode arrays were permanently implanted, we did sometimes record units with distinctly different response properties from a single electrode during different recording sessions, suggesting that the electrodes gradually shifted position in the cortex. Therefore, we set a criterion to decide whether single units collected from the same electrode on different days corresponded to the same or different units. We used the response properties to target $\mathrm{ON}$ and OFF to categorize the activity from one electrode on different days as same or different. If the recordings from one electrode in two consecutive sessions showed a unit with a similar response profile, defined as the response properties to target $\mathrm{ON}$, target OFF, and the shape of the response, then these units were considered as one cell in the analysis. Since response properties can change over time (Bichot et al., 1996) and two nearby cells can share the same response properties, this method is prone to error. But even if a small percentage of cells were assumed to be one cell when they were actually different, the overall conclusions would not be affected, although the total number of units reported would be larger.
Data analysis. For each single unit, activity was smoothed using a $40 \mathrm{~ms}$ boxcar filter and the average firing rate and SD were calculated for the period between 2 and $1 \mathrm{~s}$ preceding lever presses. A peristimulus time histogram (PSTH) was calculated for real and illusory transitions. If the PSTH showed an increase or decrease in firing rate in the period between 700 and $200 \mathrm{~ms}$ before lever press that was larger or smaller than 3 SDs from the mean firing rate, the unit was considered to have a significant modulation. Response latencies for individual cells to stimulus ON and OFF were calculated as the point in time when activity exceeded 2 SDs from the mean after target onset or offset.

For the population analysis, we computed a running firing rate using a $200 \mathrm{~ms}$ window on the unfiltered data. Then the average pre-lever press histogram was calculated and smoothed with a 3 ms Gaussian filter. All cells with same sign response profile (significant increase or decrease with respect to baseline) were averaged together to obtain the overall population average.

To calculate the modulation indices for each cell, we generated a distribution of firing rates preceding 10,000 randomly chosen lever press times from all trials (between 700 and $200 \mathrm{~ms}$ before lever press), excluding $1 \mathrm{~s}$ before and after real target transitions. Then a receiver operating characteristic (ROC) curve was created with the distribution of random target transitions versus the distribution of real target transitions. The ROC curve plots the probability for every possible firing rate (maximum firing rate normalized to 1) that the response came from the random versus the real distribution (Green and Swets, 1966). If the two distributions are the same for every possible firing rate, the probability of random versus real will be 0.5 , so the ROC curve will be a diagonal. The modulation index is the area under the curve, which provides an estimate of the separation of the real and random distributions. An index of 0.5 indicates that the two distributions are not different, whereas an index larger than 0.5 indicates that the distribution of firing rates preceding real lever presses is larger than expected by chance, and an index smaller than 0.5 indicates that the distribution of firing rates preceding real lever presses is smaller than expected by chance. Significance of the indices was calculated using bootstrap analysis to extract 1000 random groups of lever presses, each containing the same number of lever presses as the number of illusory transitions in a given recording session. An index was considered significant if the value was larger or smaller than $95 \%$ of the random sample indices.

\section{Results}

Great care was taken to ensure that the monkeys faithfully reported their perceptual state (Leopold et al., 2003). Random lever presses were discouraged by rewarding the monkeys for accuracy and not number of presses. To evaluate the effects of stimulus manipulation on the rate of disappearance, we tested the effect of changing mask speed and target size on the rate of disappearance in separate behavioral sessions. Each monkey underwent five behavioral sessions where mask speed and target size were manipulated. Two mask speeds ( 0.12 and 0.25 revolutions per second) and two target sizes $\left(0.6 \times 0.6^{\circ}\right.$ and $\left.0.8 \times 0.8^{\circ}\right)$ were tested. The monkeys' psychophysical performance followed the same trends as that of human observers. Human observers report more frequent target disappearances for faster moving distracters or smaller targets (Bonneh et al., 2001); monkeys reported likewise (Fig. 1A; supplemental Table S1, available at www.jneurosci.org as supplemental material). While performing the task, monkeys were required to respond in a time window between $150 \mathrm{~ms}$ and $1000 \mathrm{~ms}$ after the target transition. If their response was too fast or too slow, the trial was aborted. Within these temporal bounds, reaction times ranged between $270 \mathrm{~ms}$ to $980 \mathrm{~ms}$, with mean reaction times for target $\mathrm{ON}$ of $614 \mathrm{~ms}$ (monkey 1, $683 \mathrm{~ms}$; monkey 2, $545 \mathrm{~ms}$ ) and target OFF of $491 \mathrm{~ms}$ (monkey 1, $460 \mathrm{~ms}$; monkey 2, $522 \mathrm{~ms}$ ).

In addition, eye movements in humans have an effect on illusory transitions, with a lower frequency of microsaccades preceding illusory disappearances compared with illusory reappearances (Hsieh 
A

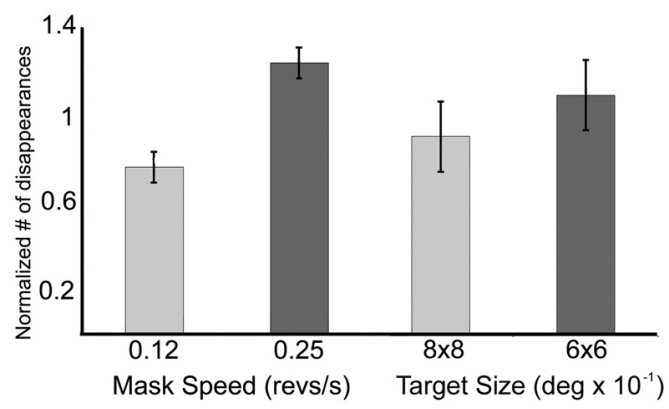

B Small eye-movements prior to lever press

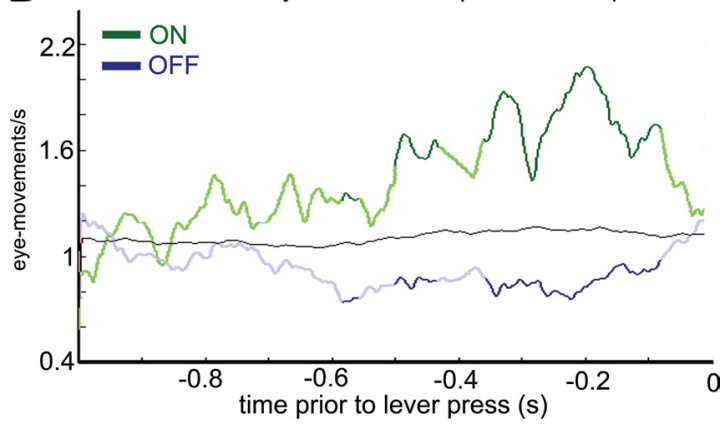

Figure 1. Behavioral results. $\boldsymbol{A}$, Number of illusory disappearances the two monkeys reported under different mask speeds (left) and target size (target diameter; right). Values are normalized to the average of each monkey over each condition (for raw values, see supplemental Table S1, available at www.jneurosci.org as supplemental material). $\boldsymbol{B}$, Average number of small eye movements per second, averaged over every trial for both monkeys, preceding reported illusory transitions for target disappearances (blue) and appearances (green). Values are the average eye-movement rate during $200 \mathrm{~ms}$ around each time point. Dark green and dark blue indicate a significant difference between the $0 \mathrm{~N}$ and $\mathrm{OFF}$ traces, as assessed by a lever randomization test ( 1000 iterations; $p<0.1$ ) between the rate of small eye movements preceding appearances versus disappearances. Black, Mean number of small eye movements as assessed by randomization of lever presses (1000 repetitions of random distributions of lever presses).

and Tse, 2009). Similarly, we found that the monkeys showed a lower frequency of small eye movements before illusory disappearances compared with illusory reappearances (Fig. $1 \mathrm{~B}$ ).

We recorded from 220 single units in FEF of two monkeys while they reported the appearance and disappearance of a target surrounded by moving blue crosses. The results from the two monkeys were virtually identical; therefore they were combined for analysis. We measured the mean and SD of the firing rate for the interval 2 to $1 \mathrm{~s}$ preceding the lever press and, using this measurement as an indication of baseline activity (real and illusory combined), calculated the number of cells where activity in response to real transitions exceeded 3 SDs from the mean in the period between 700 and $200 \mathrm{~ms}$ before lever press. One hundred and eighty-nine single units showed significant responses time locked to lever presses in response to either the real onset or the real offset of a visual target. The responses could be either an increase or a decrease in the firing rate and could be elicited by either target onset, offset, or both (Sato and Schall, 2001). Response latencies to real target transitions for cells that increased their firing rate after stimulus onset or offset were $73 \mathrm{~ms}$ for stimulus onset $(n=137$; range, $38-122)$ and $70 \mathrm{~ms}$ for stimulus offset ( $n=47$; range, $31-137$ ). Since some of the cells recorded also showed an increase in activity that was time locked to the lever press, all analyses presented here were done on activity preceding the lever press by at least $200 \mathrm{~ms}$. Furthermore, analyses were conducted only on trials where no small eye movements or blinks occurred in the period between 700 and 200 ms preceding lever press.
To compare real and illusory transitions, we first looked at disappearances only, given that the stimulus preceding both real and illusory disappearances is identical (target present), whereas the stimulus preceding real and illusory appearances is different (target absent for real and present for illusory). Of the 189 cells with significant responses to real target transitions (that is, cells whose receptive field overlapped the target), 85 were target-off responsive, with activity modulation to real target disappearances; 67 of these 85 cells (79\%) also showed a significant modulation of activity preceding reports of illusory disappearances (Fig. 2 ). Furthermore, the sign of the response to the illusory transitions was always in the same direction as for real transitions; in other words, if a cell increased its firing rate in response to a real target disappearance, it also increased its firing rate before the monkey's report of an illusory disappearance.

Population averages of all the units with the same sign of responses to real target transitions showed that activity preceding lever presses to illusory transitions was remarkably similar to the activity preceding real transitions in direction, magnitude, and timing (Fig. 3). Thompson and Schall (2000) reported that late responses in FEF, which start $\sim 50 \mathrm{~ms}$ after the initial response and slowly increase over hundreds of milliseconds, correlate well with perceptual report of monkeys during backward masking (Thompson and Schall, 2000). We cannot measure directly the latency for the illusory transition activity, since there is no real visual stimulus transition, but we can directly compare the timing of the illusory and real activities, and we can measure the response latencies to the real transitions. If the activations we observe here that precede illusory transitions correspond to the late activations reported by Thompson and Schall (2000), we would expect the peak activations preceding illusory transitions to occur later than the peak activations that precede real transitions. To test whether a delay in neuronal responses before illusory reports would elicit observable changes in these average plots, we performed the same analysis on modeled data from an artificial spiking model while manipulating different parameters to mimic possible delayed response scenarios. We focused on cells that increased their activity in response to the onset of the target (Fig. 3, top left). We first generated 137 artificial cells by randomly assigning spikes in a $3 \mathrm{~s}$ time window. We then added the response to the target onset in each model cell. After defining a point in time when the target comes on in the model, we chose for each cell a response latency (a random time between 50 and 150 ms, parameters extracted from our recorded cells), after which an increase in firing rate occurs, where a target firing rate was chosen randomly (from 2 - to 50 -fold increase) and spikes were added to match the target firing rate within an interval between the chosen latency and a latter point in time. This interval defines the response duration (between 10 and $100 \mathrm{~ms}$ ). After this step we had 137 model cells, each with a different baseline firing rate, different increases in response to a target, different response latencies, and different response durations to the target, all mimicking the cells we recorded. To generate preresponse histograms (as in Fig. 3), we selected reaction times from the distribution of real reaction times (between 250 and $850 \mathrm{~ms}$ ) and averaged the responses of all 137 cells aligned to response time. To illustrate the fit of the model, we compared neuronal activity preceding lever ON behavioral responses for both real and model activity (supplemental Fig. S2, available at www.jneurosci.org as supplemental material). We then manipulated the timing of the model cells' responses, showing that delays as small as $50 \mathrm{~ms}$ are evident in the population response times (supplemental Fig. S3, available at www.jneurosci.org as supplemental material). 
A
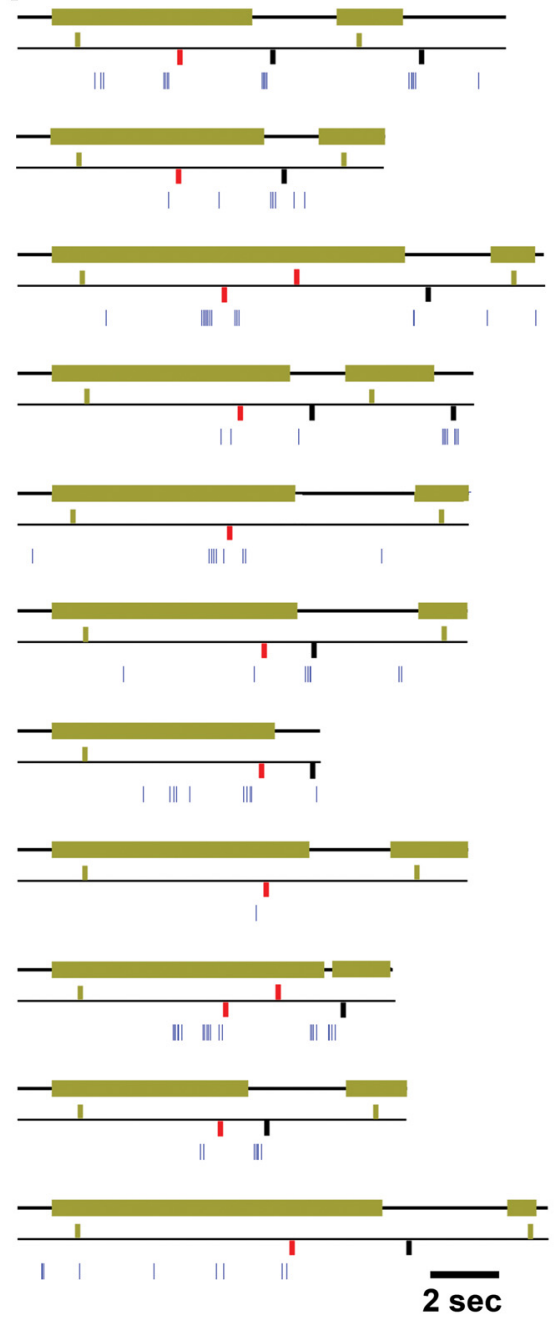
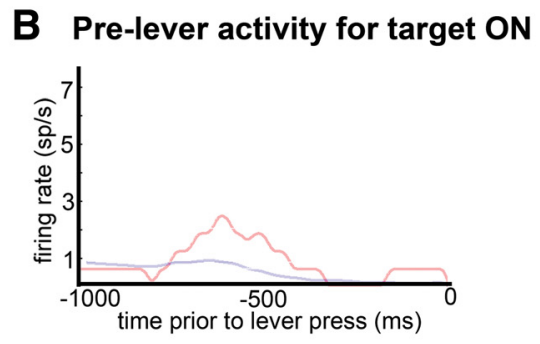

Pre-lever activity for target OFF
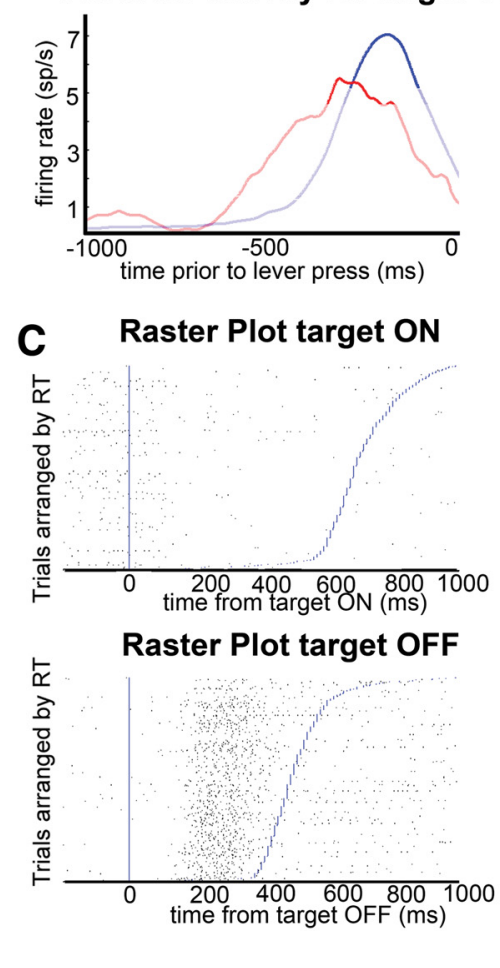

Figure 2. Sample cell in FEF. A, Activity of a single cell in FEF during the MIB task. Eleven individual trials are represented. The top trace of each trial represents the target; thick green line represents target $\mathrm{ON}$, and thin black line represents target $0 \mathrm{FF}$. The middle trace represents lever presses, bars above the black trace represent a lever press signaling the appearance of the target, and bars below the black trace represent a lever press signaling the disappearance of the target. Green, black, and red bars indicate real appearances, real disappearances, and illusory transitions, respectively. The lower trace (blue) represents the spiking activity of the neuron; each tick corresponds to one action potential. $\boldsymbol{B}$, Average activity of cell shown in $\boldsymbol{A}$ preceding lever responses indicating target $0 \mathrm{~N}$ (top) and target $0 \mathrm{FF}$ (bottom). Blue and red traces indicate activity preceding real and illusory transitions, respectively. Dark traces indicate activity exceeding 2 SDs from the mean. $C$, Raster plot aligned to target $0 \mathrm{~N}$ (top) and OFF (bottom) at time 0 (blue vertical line), and sorted by reaction time, indicated by blue lines.

We observe that the peak activations for both real and illusory transitions overlap (on average, the peak activation for illusory transitions preceded that of real transitions by $34 \mathrm{~ms}$ ) (Fig. 3). Since the latencies for real target transitions were $\sim 70 \mathrm{~ms}$, the similarity in time course between illusory and real responses, when aligned to lever press, suggests that the illusory responses must also occur quite early in the process of perceiving an illusory transition.

To compare the illusory and real transition responses to both target appearances and disappearances, we used an ROC analysis to calculate a modulation index for each cell; this allowed us to quantify the correlation between the physical stimulus and perceptual state (see Materials and Methods). Figure 4 shows the modulation indices for real versus illusory transitions for all 189 cells with significant visual responses to real target transitions. Changes in activity preceding reports of illusory transitions were strongly correlated with changes in activity preceding reports of real transitions $\left(r^{2}=0.7\right)$, indicating that the degree and direction of firing rate modulation in these cells was the same for illusory transitions as for real transitions. Since recordings were made from implanted electrode arrays and single-unit activity from different recording sessions were pooled together, we were interested to see whether the pattern observed in Figure 4 would hold in individual recording sessions. Supplemental Figure S4 (available at www.jneurosci.org as supplemental material) shows that it does, so the distribution observed in Figure 4 is not an artifact of the recording methods.

Activity in the FEF is correlated with intentional eye movements (Schall, 2002). Our task required monkeys to maintain fixation within a window; however, small saccades could occur within this window. To avoid eye-movement effects on FEF activity, we only analyzed trials where no measurable eye movements or blinks preceded lever presses. However, we also considered the possibility that the activity changes observed were the product of motor preparation to make a saccade to the target. Previous studies have shown that a correlation exists between electrically evoked saccades and the optimal saccade for eliciting presaccadic neural activity, but not postsaccadic activity (Bruce et al., 1985). We explored the possibility that the selectivity indices calculated in the ROC analysis might be related to the distance between electrically induced saccade location and target location. We observed no relationship between electrically evoked saccades and ROC values in the subset of neurons analyzed (supplemental Fig. S5, available at www.jneurosci.org as supplemental material). To explore the relationship between perisaccadic activity and visual response properties, a subset of cells were categorized as having presaccadic, postsaccadic, or no-saccadic activity using a task where monkeys had to saccade to a stable visual target. We saw no correlation between ROC values and presaccadic, postsaccadic, or no-saccade related activity (supplemental Fig. S6, available at www. jneurosci.org as supplemental material). Thus, the activity changes observed are unlikely to be the result of the generation of small eye movements or motor preparation to saccade to the target.

\section{Discussion}

At present, we can only speculate on the source of neuronal activity preceding illusory target transition reports. They could reflect a signal coming from other prefrontal cortical areas (Lumer et al., 1998), they could be the product of a faulty readout of the activity coming from early visual areas (Libedinsky et al., 2009), or they could be inherited from visual areas that project to FEF and that already correlate with perceptual state. With regard to this last possibility, one source of fast visual input to FEF is V1 to MT to FEF (Maunsell and van Essen, 1983). We have previously 
shown that activity in V1 does not correlate with perception during MIB but is reduced by MIB background motion (Libedinsky et al., 2009). Therefore, it is possible that activity in area MT correlates with perceptual detection of the target and relays this information to FEF. Previous studies have looked at the role of MT in visual discrimination of motion, but its role in perceptual detection remains unexplored (Bradley et al., 1998; Dodd et al., 2001; Grunewald et al., 2002; Schall, 2002; Williams et al., 2003).

FEF has traditionally been considered to be involved in the generation of purposive saccadic eye movements (Schall, 2002). We provide evidence that activity changes observed during illusory transitions cannot be explained by saccade generation or planning. More recently, it has been suggested that FEF plays an important role in the deployment of top-down attention (Armstrong et al., 2006; Gregoriou et al., 2009). The task used in this study required constant attention to the location of the target and we only analyzed trials where no eye movements occurred before the report of an illusory disappearance, because eye movements might reflect changes in covert attention (Martinez-Conde et al., 2009). Therefore, the activity changes observed before illusory transitions cannot easily be explained by changes in top-down attention either.

Only two studies have looked directly at the link between single-cell activity in FEF and visual perception. Thompson and Schall (1999), using a backward-masking paradigm, showed that FEF neurons show early responses to both detected and undetected targets and a more delayed response only to detected targets and false alarms (Thompson and Schall, 2000). They found that initial responses to detected targets are slightly stronger (1-2 spikes per trial) than to undetected targets (Thompson and Schall, 2000). In other words, they saw a weak correlation between early FEF activity and the reports of the subjects, which seems at odds with our finding of a strong correlation between early FEF activity and perceptual report, with average activity preceding reports of illusory transitions being more than half the magnitude of responses to real transitions (Fig. 3 ). We believe that the differences between the two tasks, backwardmasking and MIB, can explain these differences. In contrast to backward masking, which produces near-threshold perception, MIB produces a strong and clear perceptual dichotomy. Furthermore, in our experimental design, behavioral responses were not prompted (they were self-motivated), whereas during the backward-masking task, responses were prompted by the experimenter in a twoalternative, forced-choice task. The perceptual report is bimodal during both tasks. Perceptual experience is not bimodal in the backward-masking task, but it is close to bimodal in the MIB task. Therefore, during backward-masking, small differences in perception and decision criterion become amplified in reporting by a twoalternative forced choice. Therefore, even if FEF activity is strongly related to the perceptual state of the subject, as we propose, we would nevertheless expect a weak correlation between the initial neural response and perceptual report during backward masking, as Thompson and Schall $(1999,2000)$ found.
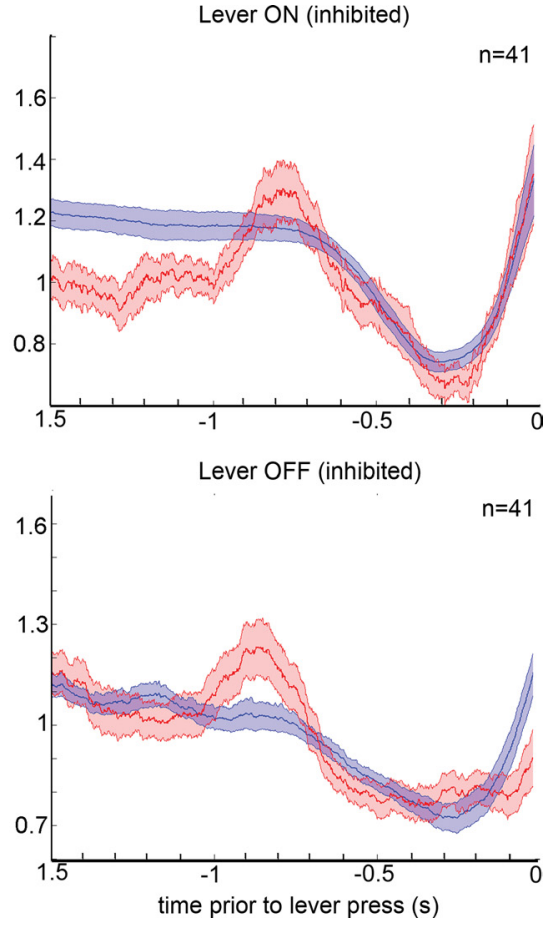

$=41$

OFF (excited)

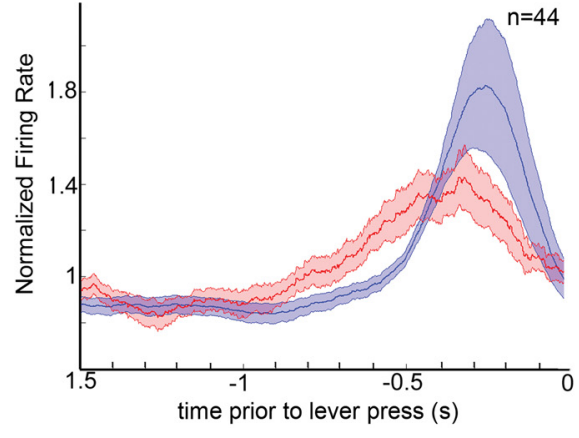
.

Figure 3. Population average. Average firing rate preceding lever presses signaling real (blue) and illusory (red) transitions, normalized to the average firing rate for each cell. The population was divided between cells that were excited (left column) or inhibited (right column) by target onset (top row) or target offset (bottom row). Thick lines represent the average, and shaded area represents 1 SE from the mean.

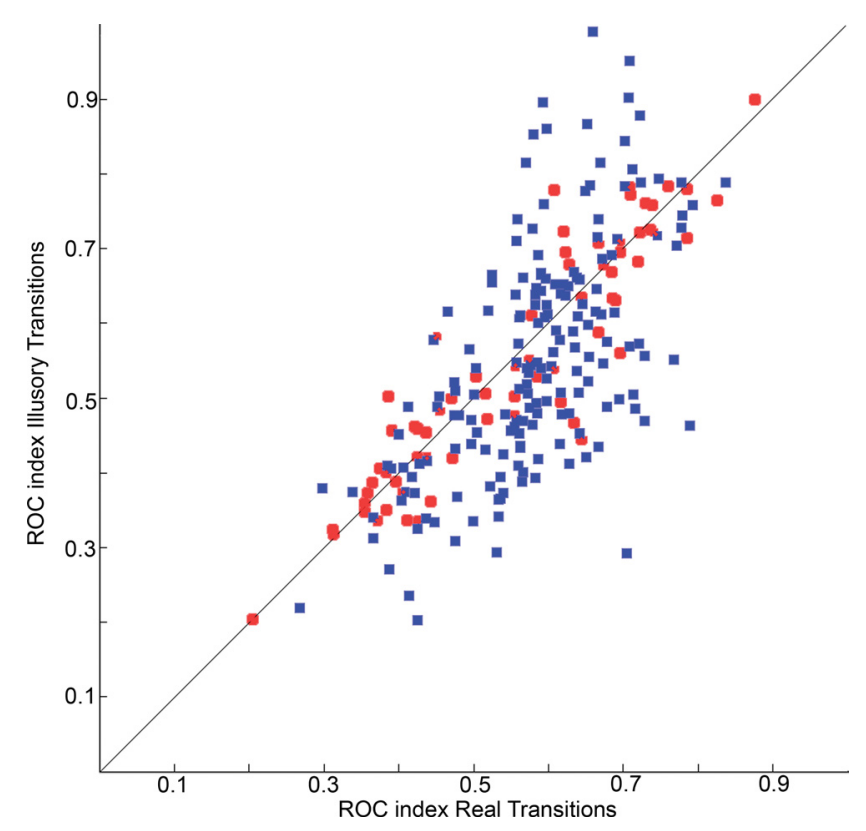

Figure 4. Modulation indices for the population. Modulation index for real versus illusory transitions for Target ON (blue squares) and Target OFF (red circles) for the whole population of FEF cells with significant responses to real target transitions.

Functional imaging studies have shown that early visual areas (V1, V2, V4, and MT) activate in association with illusory disappearances during MIB (Donner et al., 2008; Schölvinck et al., 2009). More specifically, these studies show neural signatures associated exclusively with illusory disappearances but not present in real ones. Since we found no difference in FEF activations between illusory and real disappearances, our results suggest that FEF is not 
the source of these illusory-specific disappearance activations and, likewise, that these illusory-specific disappearance activations cannot be the source of the activations we observed in FEF.

The neuronal mechanisms that lead to a percept during normal viewing must be somehow different from those that lead to the same percept during ambiguous viewing, i.e., MIB or binocular rivalry (Logothetis and Schall, 1989; Leopold and Logothetis, 1996; Sheinberg and Logothetis, 1997; Libedinsky et al., 2009; Lumer et al., 1998). However, it is assumed that both mechanisms converge at a certain point and then follow the same path to perception and action. This point of convergence and subsequent steps are candidates to form part of the neuronal correlates of perception (Koch, 2004). In the present study, we have identified FEF as an area with quite early activity that correlates with perceptual state, thus making FEF a potential point of such a convergence. Furthermore, it is possible that other areas with activities that correlate with perceptual state are subject to direct or indirect influence from FEF. For example, Logothetis and colleagues, using binocular rivalry, have shown that activity in early visual areas correlates mostly with visual stimulus, whereas activity in late visual areas correlates mostly with perceptual report (Logothetis and Schall, 1989; Leopold and Logothetis, 1996; Sheinberg and Logothetis, 1997). In light of our results, we could interpret the results obtained by Logothetis and colleagues as a consequence of the influence of FEF over visual areas. This interpretation fits well with the observation that higher visual areas (such as IT) show higher correlation with perception than lower visual areas (such as V4 or V1) do, since higher visual areas receive stronger projections from FEF than lower visual areas (Stanton et al., 1995). Future research could address this possibility.

As previously mentioned, FEF is involved in the control of voluntary eye movements (Schall, 2002). Others have shown that microstimulation in FEF can be perceived (Murphey and Maunsell, 2008) without directly examining the role of FEF in visual perception. We now provide evidence that early firing in FEF correlates with visual perception. Notably, visual perception fades in the absence of eye movements (Ditchburn and Ginsborg, 1952; Riggs et al., 1953) and the main source of visual change in monkeys and humans are eye movements. We suggest that this convergence of visual and oculomotor roles of FEF is not a coincidence. Whether this relationship holds in other sensory modalities could offer a clue about fundamental principles of sensory perception (O'Regan and Noë, 2001).

\section{References}

Armstrong KM, Fitzgerald JK, Moore T (2006) Changes in visual receptive fields with microstimulation of frontal cortex. Neuron 50:791-798.

Asaad WF, Rainer G, Miller EK (2000) Task-specific neural activity in the primate prefrontal cortex. J Neurophysiol 84:451-459.

Bichot NP, Schall JD, Thompson KG (1996) Visual feature selectivity in frontal eye fields induced by experience in mature macaques. Nature 381:697-699.

Bonneh YS, Cooperman A, Sagi D (2001) Motion-induced blindness in normal observers. Nature 411:798-801.

Bradley DC, Chang GC, Andersen RA (1998) Encoding of threedimensional structure-from-motion by primate area MT neurons. Nature 392:714-717.

Bruce CJ, Goldberg ME (1985) Primate frontal eye fields. I. Single neurons discharging before saccades. J Neurophysiol 53:603-635.

Bruce CJ, Goldberg ME, Bushnell MC, Stanton GB (1985) Primate frontal eye fields. II. Physiological and anatomical correlates of electrically evoked eye movements. J Neurophysiol 54:714-734.

Ditchburn RW, Ginsborg BL (1952) Vision with a stabilized retinal image. Nature 170:36-37.

Dodd JV, Krug K, Cumming BG, Parker AJ (2001) Perceptually bistable three-dimensional figures evoke high choice probabilities in cortical area MT. J Neurosci 21:4809-4821.

Donner TH, Sagi D, Bonneh YS, Heeger DJ (2008) Opposite neural signatures of motion-induced blindness in human dorsal and ventral visual cortex. J Neurosci 28:10298-10310.

Green DM, Swets JA (1966) Signal detection theory and psychophysics. New York: Wiley.

Gregoriou GG, Gotts SJ, Zhou H, Desimone R (2009) High-frequency, long-range coupling between prefrontal and visual cortex during attention. Science 324:1207-1210.

Grunewald A, Bradley DC, Andersen RA (2002) Neural correlates of structure-from-motion perception in macaque V1 and MT. J Neurosci 22:6195-6207.

Hsieh PJ, Tse PU (2009) Microsaccade rate varies with subjective visibility during motion-induced blindness. PLoS One 4:e5163.

Kirchner H, Barbeau EJ, Thorpe SJ, Régis J, Liégeois-Chauvel C (2009) Ultra-rapid sensory responses in human frontal eye field region. J Neurosci 29:7599-7606.

Koch C (2004) Quest for consciousness: a neurobiological approach. Englewood, CO: Roberts and Co.

Leopold DA, Logothetis NK (1996) Activity changes in early visual cortex reflect monkeys' percepts during binocular rivalry. Nature 379:549-553.

Leopold DA, Maier A, Logothetis NK (2003) Measuring subjective visual perception in the nonhuman primate. J Consc Stud 10:115-130.

Libedinsky C, Savage T, Livingstone M (2009) Perceptual and physiological evidence for a role for early visual areas in motion-induced blindness. J Vis 9:1-10.

Logothetis NK, Schall JD (1989) Neuronal correlates of subjective visual perception. Science 245:761-763.

Lumer ED, Friston KJ, Rees G (1998) Neural correlates of perceptual rivalry in the human brain. Science 280:1930-1934.

Martinez-Conde S, Macknik SL, Troncoso XG, HubelDH (2009) Microsaccades: a neurophysiological analysis. Trends Neurosci 32:463-475.

Maunsell JH, van Essen DC (1983) The connections of the middle temporal visual area (MT) and their relationship to a cortical hierarchy in the macaque monkey. J Neurosci 3:2563-2586.

Murphey DK, Maunsell JH (2008) Electrical microstimulation thresholds for behavioral detection and saccades in monkey frontal eye fields. Proc Natl Acad Sci U S A 105:7315-7320.

O’Regan JK, Noë A (2001) A sensorimotor account of vision and visual consciousness. Behav Brain Sci 24:939-973.

Pouget P, Emeric EE, Stuphorn V, Reis K, Schall JD (2005) Chronometry of visual responses in frontal eye field, supplementary eye field, and anterior cingulate cortex. J Neurophysiol 94:2086-2092.

Riggs LA, Ratliff F, Cornsweet JC, Cornsweet TN (1953) The disappearance of steadily fixated visual test objects. J Opt Soc Am 43:495-501.

Sato T, Schall JD (2001) Pre-excitatory pause in frontal eye field responses. Exp Brain Res 139:53-58.

Schall JD (2002) The neural selection and control of saccades by the frontal eye field. Philos Trans R Soc Lond B Biol Sci 357:1073-1082.

Schmolesky MT, Wang Y, Hanes DP, Thompson KG, Leutgeb S, Schall JD, Leventhal AG (1998) Signal timing across the macaque visual system. J Neurophysiol 79:3272-3278.

Schölvinck ML, Rees G (2010) Neural correlates of motion-induced blindness in the human brain. J Cogn Neurosci 22:1235-1243.

Sheinberg DL, Logothetis NK (1997) The role of temporal cortical areas in perceptual organization. Proc Natl Acad Sci U S A 94:3408-3413.

Stanton GB, Bruce CJ, Goldberg ME (1995) Topography of projections to posterior cortical areas from macaque frontal eye fields. J Comp Neurol 353:291-305.

Thompson KG, Schall JD (1999) The detection of visual signals by macaque frontal eye field during masking. Nat Neurosci 2:283-288.

Thompson KG, Schall JD (2000) Antecedents and correlates of visual detection and awareness in macaque prefrontal cortex. Vision Res 40:15231538.

Thompson KG, Hanes DP, Bichot NP, Schall JD (1996) Perceptual and motor processing stages identified in the activity of macaque frontal eye field neurons during visual search. J Neurophysiol 76:4040-4055.

Williams ZM, Elfar JC, Eskandar EN, Toth LJ, Assad JA (2003) Parietal activity and the perceived direction of ambiguous apparent motion. Nat Neurosci 6:616-623. 